\title{
A spatially resolved relaxation method for pLTE plasma diagnostics in free-burning arcs
}

\author{
G Kühn ${ }^{1,2}$ and M Kock ${ }^{1}$ \\ ${ }^{1}$ Institut für Atom- und Molekülphysik, Abteilung Plasmaphysik, Universität Hannover, \\ Callinstraße 38, D-30167 Hannover, Germany \\ ${ }^{2}$ Institut für Gravitationsphysik, Universität Hannover, Callinstraße 38, D-30167 Hannover, \\ Germany
}

E-mail: gerrit@pmp.uni-hannover.de

Received 30 November 2005, in final form 24 February 2006

Published 18 May 2006

Online at stacks.iop.org/JPhysD/39/2401

\begin{abstract}
We report on a relaxation method to determine the electron-to-gas temperature ratio in the near-cathode region of a low-current free-burning arc operated in argon under atmospheric pressure. We used an intensified CCD camera to record the relaxation process after a fast power-interruption of the discharge with a time resolution better than $1 \mu \mathrm{s}$. For the CCD images we applied an ABEL inversion procedure yielding 3D information about the temperature ratio $\beta:=T_{\mathrm{e}} / T_{\mathrm{g}}$ with a spatial resolution of $50 \mu \mathrm{m}$. We deduced spatially resolved values of $\beta$ from the relaxation experiment using independently measured values of $T_{\mathrm{e}}$ and $n_{\mathrm{e}}$, so we could determine all free parameters in the partial LTE model.
\end{abstract}

(Some figures in this article are in colour only in the electronic version)

\section{Introduction}

In previous papers [1-3] we presented results from diagnostics in the near-cathode region of a low-current free-burning arc in argon. We found four phenomenologically distinguishable discharge modes and concentrated our work on two of them, namely the 'diffuse mode' and the 'blue-core mode'. In particular, the latter mode showed considerable deviations from local thermodynamic equilibrium (LTE) in spite of high temperatures (above $20000 \mathrm{~K}$ ) and high electron densities (up to $2 \times 10^{23} \mathrm{~m}^{-3}$ ). However, we were not able to access the cathode closer than about $1 \mathrm{~mm}$ with laser-based techniques (Rayleigh and Thomson scattering) due to the large intensity of stray light. The diagnostic technique applicable in this region is emission spectroscopy.

Emission spectroscopy employing modern CCD detectors is a reliable source of data. However, the deviations from LTE cannot be described in terms of the partial LTE (pLTE) model traditionally used in cases such as this by applying solely emission spectroscopy. The reason for this shortcoming is that there is no easy way to determine the gas temperature $T_{\mathrm{g}}$ spectroscopically. The broadening of the emitted lines is dominated by the Stark effect, leaving the Doppler profile (and hence the kinetic temperature) unaccessible.

In this paper we use a relaxation method to determine the difference between the electron temperature $T_{\mathrm{e}}$ and the gas temperature $T_{\mathrm{g}}$ in terms of the temperature ratio $\beta:=T_{\mathrm{e}} / T_{\mathrm{g}}$ for the diffuse mode. Although, the relaxation method has been used for this purpose before in the literature, this is (to our knowledge) the first time it is applied with a fast intensified CCD camera as a detector. Our setup allows spatially resolved measurements (similar to the setup for classical emission spectroscopy) and leads to 3D spatially resolved data via an Abel inversion. The relaxation method turns out to be a perfect match for classical emission spectroscopy to complete the pLTE plasma model using the obtained data.

\section{Models for LTE and pLTE plasmas}

\subsection{LTE model}

We describe a LTE plasma with four basic equations [4].

(i) Dalton's law, which gives the relation between the pressure $p$, the temperature $T$ and the number densities 
$n_{a, i}$ of atoms and Density $n_{\mathrm{e}}$ of the free electrons:

$$
p=k_{\mathrm{B}} T\left(n_{\mathrm{e}}+\sum_{a=1}^{N} \sum_{i=0}^{N_{a}} n_{a, i}\right) .
$$

Here and in the following the index $a$ denotes different kinds of atoms, whereas the index $i$ denotes different ionization stages ( $i=0$ refers to a neutral atom).

(ii) The quasi-neutrality condition, which states that the number density $n_{\mathrm{e}}$ of electrons is equal to the number density of all ionic charges summed up (with $N$ being the number of different kinds of atoms and $N_{a}$ being the number of ionization stages to be taken into account):

$$
n_{\mathrm{e}}=\sum_{a=1}^{N} \sum_{i=1}^{N_{a}} i \cdot n_{a, i} .
$$

(iii) Saha equations, which relate $n_{\mathrm{e}}$ with the ionization stage densities $n_{a, i}$ and $n_{a, i+1}$ :

$$
\begin{aligned}
S_{a, i} & :=\frac{n_{\mathrm{e}} n_{a, i+1}}{n_{a, i}} \\
& =2 \frac{Z_{a, i+1}\left(T, n_{\mathrm{e}}\right)}{Z_{a, i}\left(T, n_{\mathrm{e}}\right)}\left(\sqrt{\frac{m_{\mathrm{e}} k_{\mathrm{B}} T}{2 \pi \hbar^{2}}}\right)^{3} \\
& \times \exp \left(-\frac{E_{a, i}^{\infty}-\Delta E_{a, i}^{\infty}}{k_{\mathrm{B}} T}\right) .
\end{aligned}
$$

Here $m_{\mathrm{e}}$ denotes the electron mass, $k_{\mathrm{B}}$ is Boltzmann's constant, $\hbar$ is Planck's constant divided by $2 \pi$ and $E_{a, i}^{\infty}$ is the ionization energy, $\Delta E_{a, i}^{\infty}$ is the lowering of the ionization energy because of the surrounding plasma and $Z_{a, i}\left(T, n_{\mathrm{e}}\right)$ is the partition function.

(iv) The Boltzmann-distribution, which describes the densities of excited states

$$
\frac{n_{a, i}^{|\nu\rangle}}{n_{a, i}}=\frac{g_{a, i}^{|\nu\rangle}}{Z_{a, i}\left(T, n_{\mathrm{e}}\right)} \exp \left(-\frac{E_{a, i}^{|\nu\rangle}}{k_{\mathrm{B}} T}\right) .
$$

Here $n_{a, i}^{|v\rangle}$ is the population density of the state $|v\rangle$ of atoms of the kind $a$ in the ionization stage $i, g_{a, i}^{|\nu\rangle}$ is the corresponding statistical weight and $E_{a, i}^{|v\rangle}$ is the excitation energy.

The term equilibrium means that all temperatures for the different processes described by (1), (3) and (4) (kinetics, ionization and excitation) are equal at any given point in space, whereas local means that this temperature may vary depending on the actual place.

In this paper we report on arc discharges operated in a pure atmosphere of argon, so that only one kind of atomic species has to be considered $(a=1)$. Thus, we omit the index $a$ from here on and denote the number of ionization stages with $N_{\mathrm{Ar}}$. We calculate the lowering of the ionization energy $\Delta E_{i}^{\infty}$ according to [5]. We calculate the partition functions $Z_{i}$ using Čebyšev approximations according to [6] and the coefficients published in [7]. This method includes Rydberg states by applying a hydrogen approximation for highly excited states. As a cutoff value we use the ionization energy diminished by $\Delta E_{i}^{\infty}$.

In the case of emission from an optically thin layer a state density $n_{i}^{|\mu\rangle}$ relates to the (spectrally integrated) line emission
Table 1. Argon line data used in this work.

\begin{tabular}{|c|c|c|}
\hline Process & Symbol & Time (s) \\
\hline Elastic collisions $\mathrm{e}^{-} \leftrightarrow \mathrm{e}^{-}$ & $\tau_{\mathrm{ee}}$ & $3 \times 10^{-12}$ \\
\hline PLTE for excited $(n \geqslant 3)$ states & $\tau_{\mathrm{ex}}$ & $2 \times 10^{-11}$ \\
\hline $\begin{array}{l}\text { Equipartition of energy by } \\
\text { elastic collisions } \mathrm{e}^{-} \leftrightarrow \dot{\mathrm{i}}\end{array}$ & $t_{\mathrm{ei}}$ & $2 \times 10^{-7}$ \\
\hline $\begin{array}{l}\text { Equipartition of energy by } \\
\text { elastic collisions } \mathrm{e}^{-} \leftrightarrow \mathrm{a}\end{array}$ & $t_{\mathrm{ea}}$ & $2 \times 10^{-6}$ \\
\hline $\begin{array}{l}\text { Collision-radiation: } \\
\text { ionization and recombination }\end{array}$ & $\tau_{\mathrm{CR}}$ & $3 \times 10^{-4}$ \\
\hline Thermal conduction & $\tau_{\text {th }}$ & $4 \times 10^{-4}$ \\
\hline Ambipolar diffusion & $\tau_{\mathrm{amb}}$ & $6 \times 10^{-4}$ \\
\hline
\end{tabular}

\begin{tabular}{lllllll}
\hline Species & $\begin{array}{l}\lambda_{\mu \nu} \\
(\AA)\end{array}$ & $\begin{array}{l}E^{|\mu\rangle} \\
\left(\mathrm{cm}^{-1}\right)\end{array}$ & $g_{\mu}$ & $\begin{array}{l}A_{\mu \nu} \\
\left(10^{6} \mathrm{~Hz}\right)\end{array}$ & $\begin{array}{l}\text { Error } \\
(\%)\end{array}$ & $\begin{array}{l}\text { Reference } \\
\text { number }\end{array}$ \\
\hline Ar I & 4510.73 & 117563 & 1 & 1.18 & 7 & {$[8]$} \\
Ar I & 4522.32 & 116660 & 3 & 0.0898 & 8 & {$[8]$} \\
Ar I & 6965.43 & 107496 & 3 & 6.39 & 5 & {$[8]$} \\
Ar I & 7147.04 & 107132 & 3 & 0.625 & 8 & {$[8]$} \\
Ar II & 4426.0 & 157673 & 6 & 81.7 & 5 & {$[9]$} \\
Ar II & 4433.84 & 194883 & 8 & 64.98 & 10 & {$[10]$} \\
\hline
\end{tabular}

Table 2. Relaxation times for processes in the atmospheric argon plasma of a cascaded arc with $\varnothing=6 \mathrm{~mm}, p=1000 \mathrm{hPa}$, $n_{\mathrm{e}}=1.5 \cdot 10^{22} \mathrm{~m}^{-3}$ and $T_{\mathrm{e}}=10000 \mathrm{~K}$. The values are taken from [45].

coefficient (LEC) as ([4])

$$
\varepsilon_{\mu \nu}=\frac{h c}{4 \pi \lambda_{\mu \nu}} n_{i}^{|\mu\rangle} A_{\mu \nu}
$$

with $h$ being Planck's constant and $c$ being the speed of light. Transition wavelengths $\lambda_{\mu \nu}$, excitation energies $E^{|\mu\rangle}$ and transition probabilities $A$ used in this paper are given in table 1.

For a given pressure and a known chemical composition of the plasma, (1)-(4) leave us one free parameter; for a calculation usually the temperature $T$ is chosen, but any other parameter (e.g. $n_{\mathrm{e}}$ ) will do, too. Our plasma composition calculation basically follows the mathematical procedure described in [11].

\section{2. pLTE model}

To reach the LTE condition, collisional processes have to dominate the plasma. We follow [13,14] to allow for deviations from LTE and to develop a model for pLTE. First we consider the energy supply chain for an arc discharge. The electric field $\vec{E}$, which provides the energy for the discharge, almost exclusively affects the free electrons, due to their high mobility. Collisional processes among free electrons are rather fast (see section 4.1, table 2), so a common kinetic temperature $T_{\mathrm{e}}$ for all electrons is established comparatively easily. But if the (elastic) collision rates between electrons and heavy particles are too low, the kinetic gas temperature $T_{\mathrm{g}}$ will markedly drop below $T_{\mathrm{e}}$.

Moreover, too low (inelastic) collision rates between electrons and atoms lead to deviations from the Boltzmann distribution for excited atomic states. All atoms of noble gases share a common structure in their energy levels. The main feature, which is also present for hydrogen, is a separation of 
the ground state from all excited states by a relatively large energy gap (see $[17,18]$ ). All excited levels of Ar I have energies above $70 \%$ of the ionization energy.

Low collision rates lead to a deviation between the ground state and the excited states in the Boltzmann distribution because the inelastic cross section for collisions with electrons is markedly lower for the ground state than for the excited states. In particular this is true for neutral atoms such as Ar I due to a lack of Coulomb interaction. Thus, the ground level of ArI is overpopulated with respect to the excited levels of Ar I, which remain in partial Saha equilibrium with the ions and the free electrons. On the other hand, we can likewise speak of an underpopulation of the excited levels with respect to the ground state. We choose the latter view for this work.

These considerations lead to the introduction of two further parameters to describe a plasma in the state of pLTE.

(i) The temperature ratio $\beta:=T_{\mathrm{e}} / T_{\mathrm{g}} \geqslant 1$ accounts for the difference between the electron temperature and the gas temperature. Thus, we modify Dalton's law (1):

$$
p=k_{\mathrm{B}}\left(n T_{\mathrm{e}}+\sum_{i=0}^{N_{\mathrm{Ar}}} n_{i} T_{\mathrm{g}}\right) .
$$

(ii) An underpopulation factor $b \leqslant 1$ accounts for the underpopulation of the excited levels of Ar I, so $b$ enters the Boltzmann distribution (4) to lower all excited state densities and the Saha-Boltzmann relation, respectively. This can be basically accomplished by replacing $g_{0}^{|\nu\rangle}$ with $b g_{0}^{|\nu\rangle}$ for all excited state densities $(\nu>0)$ of Ar I; additionally, all ion densities are underpopulated because they are in partial Saha equilibrium with the excited states of Ar I. Mathematically, it is possible to define a modified partition function $\breve{Z}_{0}$ including the $b$ factor, which leaves the rest of the equations unchanged.

The pLTE plasma model is successfully applied in the literature to describe deviations from LTE in arc discharges (see, e.g. [19-22]). The assumption of a single $b$-factor describing the deviation of all excited levels from LTE is based on the aforementioned large energy gap between the ground state and the excited states in noble gases. It is only valid if the deviations from LTE are not too large. For argon, the next energy levels beyond the ground state to show deviations from LTE are the four $4 \mathrm{~s}$ states between 93000 and $96000 \mathrm{~cm}^{-1}$. The authors of [23] have shown that the deviation factors for the next states beyond the ground state are three to four orders of magnitude smaller than the ground state factor and thus can be neglected. Their model calculations were carried out for the cathode region of a high current arc with temperatures between 12000 and $16000 \mathrm{~K}$. In addition, [24] presented experimental Ar I Boltzmann plots from the cathode region of a low-current arc for excited states. These show a (nearly) uniform excitation temperature, in contrast to the anode region, where the pLTE effects are more pronounced, so that the excited states cannot be described with a single excitation temperature. The uniform excitation temperature is in accordance with the assumption of a single $b$-factor. Thus, we think that the application of the pLTE model with a single $b$-factor as described above is justified for our experiment [25].

Because there are three free parameters (instead of only one in the LTE case), the pLTE model is cleary more difficult to determine experimentally. A single measurement is sufficient to determine the LTE model (e.g. an absolute line intensity, from which the temperature can be deduced), but we need to measure three independent quantities (corresponding to the three free parameters) in the pLTE case. To obtain an overview of the impact of the two additional parameters on the plasma model, figure 1 shows calculated LECs for various values of $T_{\mathrm{e}}$, $\beta$ and $b$ using a pseudo-colour representation for the LEC. By design of the model, the values satisfying $(\beta=1, b=1)$ correspond to the LTE values for the line. The intensity maximum for each horizontal line corresponds to the norm temperature $T_{\mathrm{N}}$ for the chosen values of $(\beta, b)$; the maximum value is called the Larenz maximum [12].

Figure 1 shows that the two additional pLTE parameters $\beta$ and $b$ have diametrically opposed influence on line emission. For a given $T_{\mathrm{e}}$ a large $\beta$ leads to a small $T_{\mathrm{g}}$ and thus via the modified Dalton law (6) to a higher density $n_{0}$ of neutral atoms. This in return increases the line emission via the Boltzmann distribution (4). On the other hand, a decreasing underpopulation factor $b$ leads to a decreasing population of the excited states and thus to a decreasing line emission. In a real pLTE plasma both $\beta$ and $b$ are different from unity and their effects on line emission can (at least partly) cancel out.

\section{Determination of plasma parameters in pLTE using emission spectroscopy}

As long as $\beta$ is unknown, Dalton's law cannot be used for the evaluation of spectroscopic data because the neutral density $n_{0}$ is unknown. This confines us to spectroscopic measurements evaluable without knowing $n_{0}$.

One possibility is the measurement of the continuous emission coefficient (CEC) $\varepsilon_{\lambda}$, which is related to $n_{\mathrm{e}}$ and $T_{\mathrm{e}}$ [26]:

$$
\varepsilon_{\lambda}=\frac{e^{6}}{12 c^{2} \varepsilon_{0}^{3} \sqrt{6 \pi^{5} m_{\mathrm{e}}^{3} k_{\mathrm{B}}}} \frac{n_{\mathrm{e}}}{\lambda^{2} \sqrt{T_{\mathrm{e}}}} \sum_{i=1}^{N_{\mathrm{Ar}}} n_{i} i^{2} \xi_{i}\left(\lambda, T_{\mathrm{e}}\right)
$$

with

$$
\begin{aligned}
\xi_{i}:= & {\left[1-\exp \left(-\frac{h c}{\lambda k_{\mathrm{B}} T_{\mathrm{e}}}\right)\right] \frac{\gamma_{i}}{Z_{i}\left(T_{\mathrm{e}}, n_{\mathrm{e}}\right)} \xi_{z}^{\mathrm{fb}}\left(\lambda, T_{\mathrm{e}}\right) } \\
& +\exp \left(-\frac{h c}{\lambda k_{\mathrm{B}} T_{\mathrm{e}}}\right) \xi_{i}^{\mathrm{ff}}\left(\lambda, T_{\mathrm{e}}\right) .
\end{aligned}
$$

The Biberman factors $\xi_{i}^{\mathrm{fb}}$ for free-bound and $\xi_{i}^{\mathrm{ff}}$ for free-free transitions of the electrons are expressed as one effective $\xi_{i}$ factor for each ionization stage $i$. For a singly ionized plasma $\left(N_{\mathrm{Ar}}=1, n_{1}=n_{\mathrm{e}}\right)$ the sum in (8) reduces to the first term $\left(n_{\mathrm{e}} \xi_{1}\right)$. For argon plasmas the simplified version of (8) is a good approximation up to about $18000 \mathrm{~K}$. Above this temperature the inclusion of the next term $\left(4 n_{2} \xi_{2}\right)$ should be considered to avoid systematic errors [27].

We chose the wavelength range between 4455 and $4456 \AA$ to measure the CEC because this region does not contain argon line emission and $\xi_{1}$ is almost independent of both wavelength and temperature. Thus, we assumed $\xi_{1}=1.86$, which is consistent with both theoretical [28] and experimental [29,30] data. The measured CEC is especially suitable to deduce the electron density $n_{\mathrm{e}}$ because $\varepsilon_{\lambda}$ in the simplified form of (8) depends on $n_{\mathrm{e}}^{2}$, but only on $\sqrt{T_{\mathrm{e}}}$ and linearly on the $\xi$ factor. 

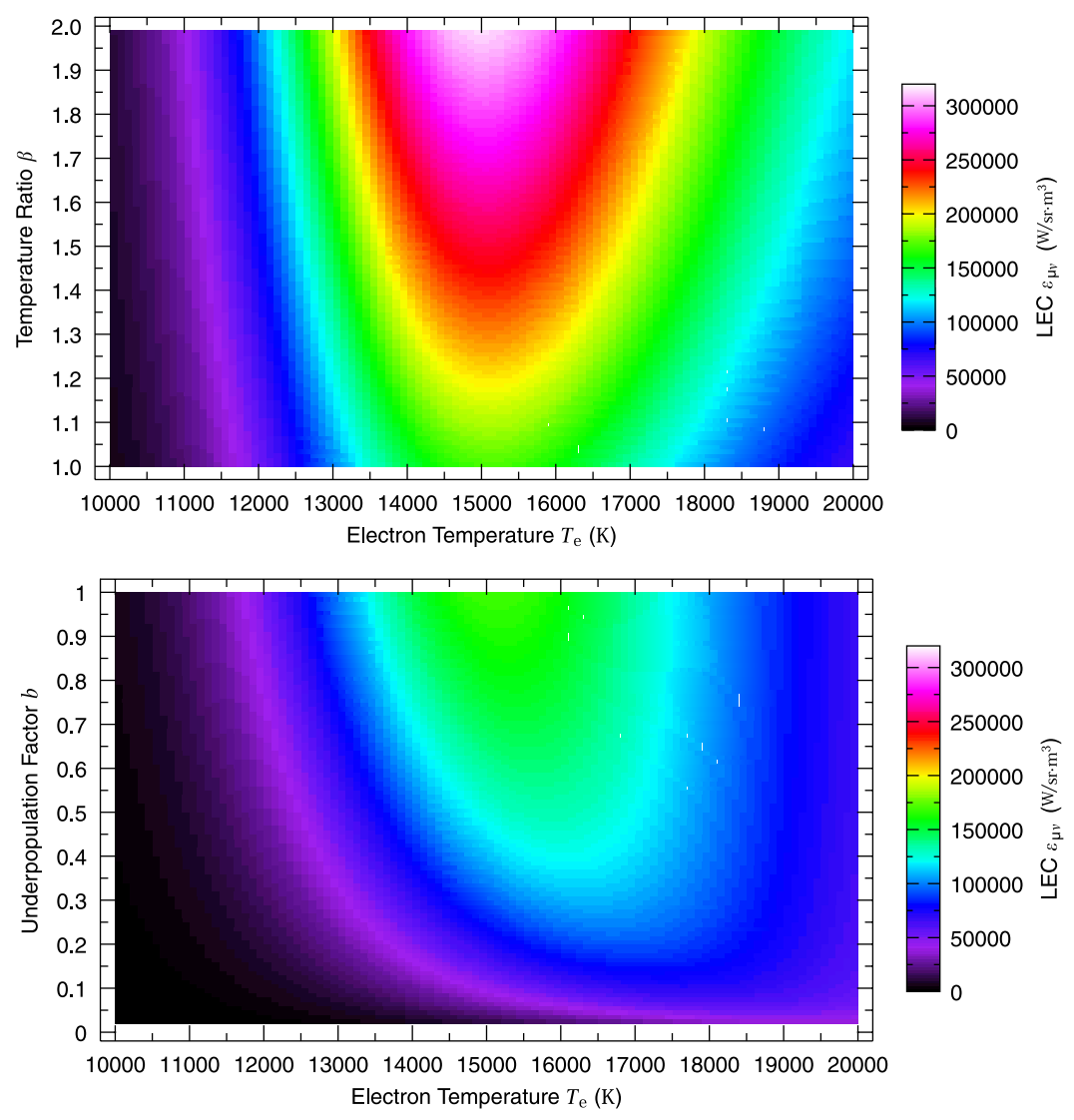

Figure 1. Calculated LECs of the Ar I line at $7147 \AA$ with $T_{\mathrm{e}}, \beta$ and $b$ as parameters. $(a)$ shows $\varepsilon_{\mu \nu}\left(T_{\mathrm{e}}, \beta, b=1\right)$, whereas $(b)$ shows $\varepsilon_{\mu \nu}\left(T_{\mathrm{e}}, \beta=1, b\right)$. This overview demonstrates that $\beta$ and $b$ have opposite effects on the line emission and that neither $T_{\mathrm{N}}$ nor the Larenz maximum are independent of the pLTE parameters.

On the other hand, we determined $T_{\mathrm{e}}$ by measuring a LEC $\varepsilon_{\mu \nu}$ and calculating the state density from (5). Further evaluation is easy for an ionic line (Ar II or Ar III) because even in pLTE we can use the Boltzmann distribution (4) for ions, and calculate the required stage densities $n_{1}$ and $n_{2}$ by applying the quasi-neutrality condition (2) and the appropriate Saha equation (3) ( $S_{2}$ does not contain any of the pLTE parameters). We preferred this procedure because the exponential factor in the Boltzmann distribution guarantees a small error in $T_{\mathrm{e}}$.

However, this procedure is limited to relatively hot plasmas producing a sufficient amount of ionic line emission. This is usually the case in the blue-core mode, but for the diffuse mode we had to use neutral lines. These could not be evaluated via the Boltzmann distribution because the stage density $n_{0}$ is unknown as mentioned above. Two other possibilities remain to determine $T_{\mathrm{e}}$ from neutral line emission.

(i) The use of a two-line method. According to the Boltzmann distribution the ratio of two excited Ar I states $|\nu\rangle$ and $|\mu\rangle$ yields

$$
\frac{n_{0}^{|\nu\rangle}}{n_{0}^{|\mu\rangle}}=\frac{g_{0}^{|\nu\rangle}}{g_{0}^{|\mu\rangle}} \exp \left(-\frac{E_{0}^{|\nu\rangle}-E_{0}^{|\mu\rangle}}{k_{\mathrm{B}} T_{\mathrm{e}}}\right),
$$

while the underpopulation factor $b$ cancels out. This concept can be extended using multiple lines to create a Boltzmann plot, which then yields $T_{\mathrm{e}}$. (ii) The use of the Saha-Boltzmann relation for the excited Ar I level and the Ar II stage density, which are in partial Saha equilibrium:

$$
\begin{aligned}
n_{0}^{|\nu\rangle} & =\frac{g_{0}^{|\nu\rangle}}{2 Z_{1}} n_{\mathrm{e}} n_{1}\left(\sqrt{\frac{2 \pi \hbar^{2}}{m_{\mathrm{e}} k_{\mathrm{B}} T_{\mathrm{e}}}}\right)^{3} \\
& \times \exp \left(\frac{E_{0}^{\infty}-\Delta E_{0}^{\infty}-E_{0}^{|v\rangle}}{k_{\mathrm{B}} T_{\mathrm{e}}}\right) .
\end{aligned}
$$

In both cases the measured LEC can be related to the state densities using (5). However, both (9) and (10) have the disadvantage that their exponential factors make the determination of $T_{\mathrm{e}}$ prone to errors arising from measurement, calibration, $A$ values, etc.

In this paper we present data obtained in the diffuse mode, using (10) for evaluation. Electron temperatures determined from ionic line emission and (4) can reach an accuracy better than $2 \%$, whereas we estimate the total temperature error for neutral line evaluation to range between $5 \%$ and $10 \%$. Our main ArI diagnostic lines were at 4511 and $4522 \AA$ (see table 1). The error for the electron density was mainly caused by uncertainties in the $\xi$-factor and measurement/calibration errors. We estimate that $n_{\mathrm{e}}$ too has an accuracy in the range $5-10 \%$. We always evaluated the measured CEC and LEC together from one acquired spectrum to obtain consistent values for $n_{\mathrm{e}}$ and $T_{\mathrm{e}}$ in one run. 


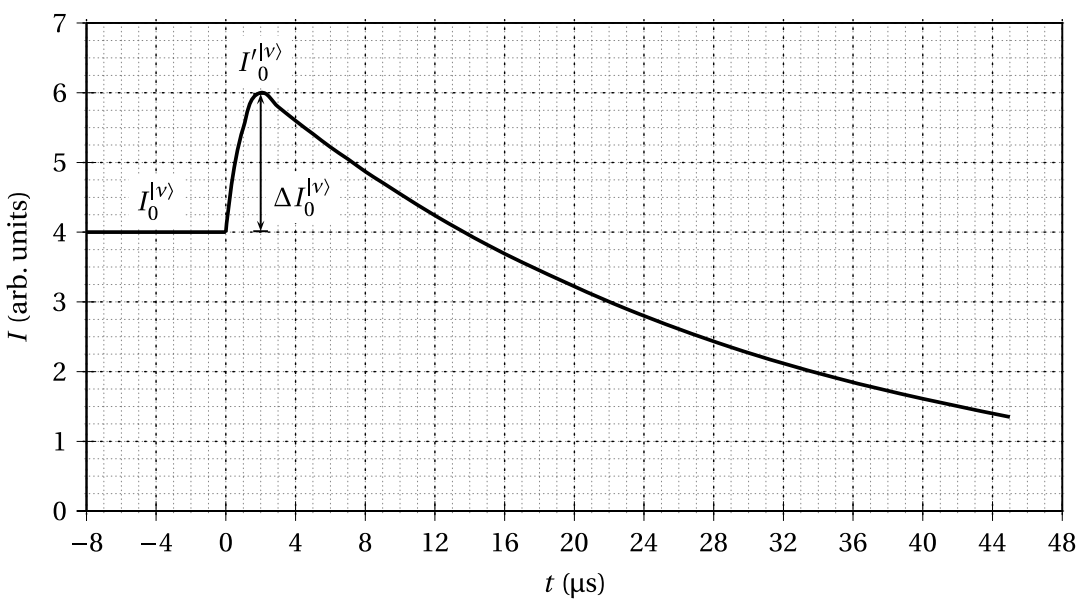

Figure 2. Schematic diagram of the Saha response of a emission line (intensity $I$ ) during power interruption in a pLTE plasma.

\section{Relaxation method}

The relaxation method is one of the few ways to gain an insight into the dynamics of non-LTE plasma processes and to determine the temperature ratio $\beta$ and hence the gas temperature $T_{\mathrm{g}}$. Basically it is a transient variant of emission spectroscopy. The transient relaxation effect, during which line and/or continuous emissions are measured, is caused by a rapid interruption of the electrical power sustaining the discharge. Therefore, we refer to this method as active emission spectroscopy. In the literature it is also known as the power-interruption technique.

Based on early works [31] the first use of the relaxation method is reported in [32]. During the following years it is used to examine the plasma of different arc discharges [3335]. Among other applications, the method is later employed on cascaded arcs [20, 22, 36-38], ICPs [39-42] and transferred $\operatorname{arcs}[43]$.

\subsection{Relaxation in LTE and pLTE plasmas}

According to [44] we describe the temporal change in the population density of an excited ArI level $n_{0}^{|\nu\rangle}$ by means of rate equations:

$$
\begin{aligned}
& \dot{n}_{0}^{|\nu\rangle}=\underbrace{n_{\mathrm{e}} \sum_{\mu} n_{0}^{|\mu\rangle} C(\mu, \nu)-n_{\mathrm{e}} \sum_{\mu} n_{0}^{|\nu\rangle} C(\nu, \mu)}_{\text {Boltzmann terms }} \\
& -n_{\mathrm{e}} n_{0}^{|\nu\rangle} S(v)+n_{\mathrm{e}}^{3} S(v) G(v) . \\
& \text { Saha terms }
\end{aligned}
$$

Here $C$ denotes collision-induced transitions (quenching) and $S$ is the coefficient for collisional ionization. $\alpha$ is the coefficient for three-body recombination, which is linked to $S$ and $G$ via the principle of detailed balancing [44]: $\alpha(v)=G(v) S(v)$ with

$$
G(\nu):=\frac{g_{0}^{|\nu\rangle}}{2 g_{1}^{|0\rangle}}\left(\sqrt{\frac{2 \pi \hbar^{2}}{m_{\mathrm{e}} k_{\mathrm{B}} T_{\mathrm{e}}}}\right)^{3} \exp \left(\frac{E_{0}^{\infty}-\Delta E_{0}^{\infty}}{k_{\mathrm{B}} T_{\mathrm{e}}}\right) .
$$

We distinguish between Saha and Boltzmann terms because the former are responsible for the Saha equilibrium, whereas the latter establish the Boltzmann distribution among the atomic states. The Saha terms are dominant for Ar I energy levels, which have excitation energies close to the ionization energy. On the other hand, the Boltzmann terms may prevail for the ground state.

If the plasma is in LTE, the state density (and thus the line emission) is constant, and its temporal derivative vanishes: $\dot{n}_{0}^{|v\rangle}=0$. A power-interruption experiment has no instant effect on the rate equations. The temperature decreases, and along with it line emission and rate coefficients decrease.

However, in pLTE the electrons lose their higher kinetic energy rather quickly due to the large collision cross-sections with excited atomic levels. Thus, their temperature $T_{\mathrm{e}}$ drops to $T_{\mathrm{g}}$ before other processes take place. For an overview of the timescales involved, we refer to table 2 . There we cite typical relaxation times originally given in [45] for an argon plasma generated in a cascaded arc, showing plasma parameters similar to our arc plasma in terms of $n_{\mathrm{e}}$ and $T_{\mathrm{e}}$. Thus, we expect the cooling process $T_{\mathrm{e}} \rightarrow T_{\mathrm{g}}$ to take about $1 \mu \mathrm{s}$. Table 2 further shows that cooling of the electrons is much faster than ionization and recombination processes. It is also faster than the usual thermal conduction and diffusion processes. So we further expect $T_{\mathrm{g}}, n_{\mathrm{e}}$ and all ion densities $n_{i}$ to be constant, while the electrons cool from $T_{\mathrm{e}}$ to $T_{\mathrm{g}}$.

\subsection{Saha response}

During the relaxation process the coefficient $S$ for collisional ionization decreases while $G$ rises according to (12). Given that the Saha terms in (11) are dominant, we infer $\dot{n}_{0}^{|\nu\rangle}>0$. This means that in a pLTE argon plasma the relaxation process following a power interruption causes an increase in the excited state densities and thus an increase (a jump) in line emission. This effect is called the Saha response [40].

Figure 2 sketches the development of the line emission during a relaxation experiment in pLTE. While the discharge is operated continuously, the line emission has a constant value $I_{0}^{|\nu\rangle}$. After interrupting the power there is a jump $\Delta I_{0}^{|\nu\rangle}$. Some microseconds later, $T_{\mathrm{e}}$ cools down to $T_{\mathrm{g}}$, and the maximum line emission $I_{0}^{|\nu\rangle}$ is reached. Having passed the maximum, the usual cooling process takes place, which causes a (slower) decrease in the signal. 


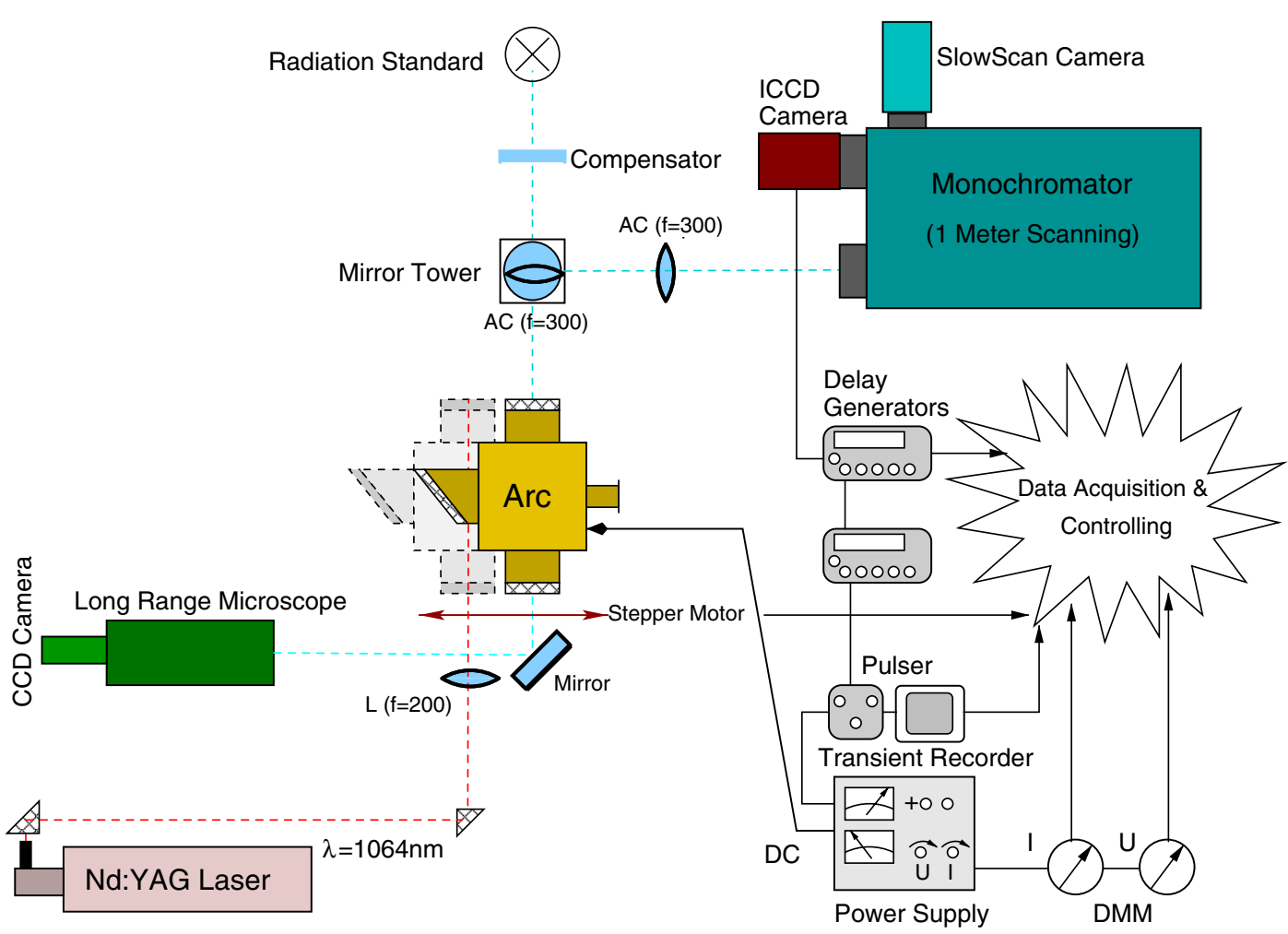

Figure 3. Overview of the experimental arrangement for passive and active optical emission spectroscopy.

Assuming partial Saha equilibrium we divide (10) for the maximum emission value reached at $T_{\mathrm{g}}$ by the continuous emission value reached at $T_{\mathrm{e}}$ and obtain

$$
\Theta:=\frac{I_{0}^{\prime|\nu\rangle}}{I_{0}^{|\nu\rangle}}=\sqrt{\beta}^{3} \exp \left(\frac{\left(E_{0}^{\infty}-\Delta E_{0}^{\infty}-E_{0}^{|\nu\rangle}\right)}{k_{\mathrm{B}} T_{\mathrm{e}}}(\beta-1)\right) .
$$

Here we also make use of the fact that the partition function for Ar II hardly depends on $T$ and that electron and ion densities are constant during the relaxation process, so $Z_{1}, n_{\mathrm{e}}$ and $n_{1}$ cancel out.

Provided that $T_{\mathrm{e}}$ and $n_{\mathrm{e}}$ have been measured before as described in section 3 , the relaxation experiment yields $\beta$ and hence $T_{\mathrm{g}}$. The determination of $\beta$ is dominated by the exponential factor in (13). $\Theta$ itself has a rather low uncertainty as it is formed by the ratio of two measured intensities, and the error propagation is further weakened by the exponential function. Thus, the main source of uncertainty for $\beta$ is $T_{\mathrm{e}}$, and we estimate the overall error to range between $7 \%$ and $15 \%$. The same measure holds for $T_{\mathrm{g}}$. We note that these are the worst case estimates for the absolute values of the measured parameters. Error sources such as uncertainty caused by the absolute calibration of the detector system shift the measured absolute values but keep their spatial behaviour in the radial or axial direction intact.

\section{Experimental setup}

\subsection{Overview}

The experimental setup shown in figure 3 was similar to the setups we used in [1-3], so we point out just the main features and enhancements here.
We constructed a new arc vessel based on a smaller and lighter cube (side length $80 \mathrm{~mm}$ ) with four optical ports. This allowed us to move the vessel with a stepper motor, so we gained better adjustability of the vessel, and we could also use the optical ports on different positions. We equipped our Questar QM-1 long-range microscope with a standard video CCD camera linked to a PC with a frame grabber card installed. This enabled us to observe and record the cathode region during the whole experiment with 25 frames per second. Thereby, we could control the stability of the discharge and (even afterwards) look for changes and instabilities. We could move the lower mirror of the mirror tower with a dc motor, increasing the reproducibility of the axial positions (also see section 5.2). The two spectroscopic camera systems were mounted on two output ports of one monochromator (selectable with a turnable mirror). This ensured that both the cameras always detected the same plasma region. We used additional delay generators, a pulser unit and a transient recorder for the relaxation experiment (also see section 5.3). The Nd: YAG laser, which has been used for Rayleigh and Thomson scattering in [1], was used to ignite the discharge (see section 5.4).

Data acquisition and controlling consisted of four PCs. We wrote the software for controlling, data acquisition and data evaluation in Java (http://java.sun.com) using the Eclipse project (http://www.eclipse.org). We adopted numerical algorithms (such as Romberg integration, Brent's method for root finding and single-value decomposition to solve systems of linear equations) from [46] and used external Java packages for image manipulation (http://rivit.cs.byu.edu/jigl/) and matrix operations (http://math.nist.gov/javanumerics/ jama/). 
A spatially resolved relaxation method for pLTE plasma diagnostics

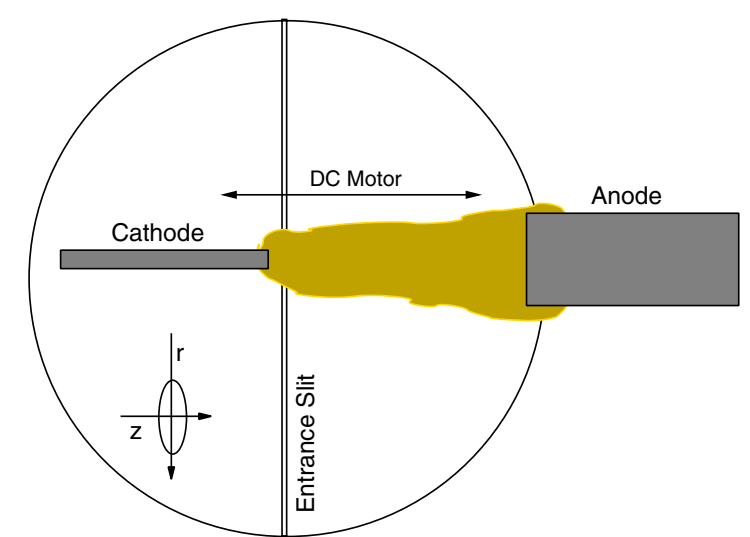

Figure 4. Image mapping of the arc plasma onto the entrance slit of the monochromator.

We reduced the number of experimental configuration parameters by creating a standard setup. The discharge was operated in argon (type 5.3 by Linde, purity better than $99.9993 \%$ ) under atmospheric pressure. The cathode was a rod of thoriated $(1.8 \% \mathrm{Th})$ tungsten $(0.6 \mathrm{~mm}$ in diameter); the anode was a rod of pure tungsten $(2.0 \mathrm{~mm}$ in diameter). We chose thoriated tungsten as cathode material to obtain measurements comparable to our previous work [1,3]. This caused a large uncertainty in the determination of the work function. On the other hand, even electrodes made of pure tungsten show a large variation in their work function, both at room temperature [47] and under arcing conditions [48]. Both electrodes had flat ends and were $30 \mathrm{~mm}$ in length. Their length outside the electrode holder was $10 \mathrm{~mm}$ in each case. The arc was operated in an upright position with the cathode below and the anode on top. The distance between the electrodes was $10 \mathrm{~mm}$. We also limited the data presented in this work to one current, namely $12.5 \mathrm{~A}$.

\subsection{Spectroscopic setup}

The image of the arc plasma was rotated by $90^{\circ}$ with the help of a mirror tower and mapped life-sized onto the entrance slit of the monochromator (see figure 4) by means of a pair of achromatic lenses. We used cylinder coordinates for the description of the positions, with $z$ denoting the axial distance from the cathode tip and $r$ denoting the radial distance from the arc centre. We set the width of the entrance slit to $50 \mu \mathrm{m}$, which defined our axial resolution. The radial resolution was defined by the size of the CCD pixels (typically $30 \mu \mathrm{m}$ ). We used a dc motor for moving the lower mirror of the mirror tower so that we could select different axial sheaths to measure.

Because we measured the plasma emission side-on, all collected spectroscopic data were integrated over the line-ofsight. We thus had to solve the corresponding Abel integral equation to achieve spatially resolved emission coefficients. For the Abel inversion we used the algorithm proposed in [49]. The procedure is based on the approximation of the unknown radial function with a Fourier series with unknown coefficients. These were determined by a least-square fit to the measured data points. In contrast to other methods of Abel inversion we neither have to use the derivative of the measured data (which leads to an amplification of noise) nor do we have to
Table 3. Technical data of the CCD camera systems.

\begin{tabular}{lll}
\hline & Slow-scan CCD & Intensified CCD \\
\hline Model & PI LN/CCD-1752-PF & RS PI-MAX-1024-E \\
Cooling & $-125^{\circ} \mathrm{C}\left(\mathrm{IN}_{2}\right)$ & $-40^{\circ} \mathrm{C}($ Peltier $)$ \\
Pixel size & $15 \mu \mathrm{m} \times 15 \mu \mathrm{m}$ & $28 \mu \mathrm{m} \times 28 \mu \mathrm{m}$ \\
Chip size & $1752 \mathrm{px} \times 532 \mathrm{px}$ & $1024 \mathrm{px} \times 256 \mathrm{px}$ \\
A/D range & $18 \mathrm{Bit}$ & $16 \mathrm{Bit}$ \\
Spectral range & $2200-11000 \AA$ & $5000-9000 \AA$ \\
Exposure time & $\geqslant 80 \mathrm{~ms}($ shutter $)$ & $\geqslant 5 \mathrm{~ns}(\mathrm{MCP})$ \\
\hline
\end{tabular}

fit the measured data before the inversion process (which may tamper with the actual data). The Fourier method provides very accurate inverted data [50]. Furthermore, we could use it as a noise filter by selecting a certain range of Fourier coefficients. Taking into account that the plasma under investigation in this work showed monotonically decreasing profiles only, which are far less prone to error for Abel inversion [51], we ensured that the inverted data had high quality.

Before the inversion of LECs, we spectrally integrated the line to improve the $S / N$ ratio and we interpolated and subtracted the neighbouring continuous radiation. For measuring CECs, we made sure that the spectral region in use was free of line emission (we usually chose the spectral range 4455-4456 $\AA$ ). In either case we made sure that the measured plasma emission preserves cylinder symmetry because asymmetry can lead to systematic errors in the Abel inversion process.

We performed the emission measurements with a $1 \mathrm{~m}$ McPherson monochromator using a grating with 1200 lines $/ \mathrm{mm}$, blazed for $500 \mathrm{~nm}$ in the first order of diffraction. The detector for passive emission spectroscopy, which we used to determine $n_{\mathrm{e}}$ and $T_{\mathrm{e}}$ as described in section 3 , was a front-illuminated slow-scan CCD camera (Princeton Instruments). The detector for the relaxation measurements was an intensified CCD camera (Roper Scientific). Some technical data of the CCD systems can be found in table 3 . The iCCD can make use of the full dynamic range with single pixels, whereas the slow-scan CCD has pixels with a linear dynamic range of about 15 Bit. Thus we usually applied $2 \times 2$ hardware binning for this system, which yielded an effective pixel size of $30 \mu \mathrm{m} \times 30 \mu \mathrm{m}$ and a dynamic range of $17 \mathrm{Bit}$.

With their high dynamic range and excellent linearity (better than 1\%), both detector systems are ideally suited to quantitative spectroscopy. In this work we used a tungsten strip lamp calibrated by the PTB (German national metrology institute) as radiation standard to measure emission coefficients on an absolute scale. The lamp was placed opposite to the arc discharge, making use of the established spectroscopic T-arrangement (the lower mirror of the mirror tower could be rotated)

\subsection{Relaxation setup}

Figure 5 shows a block diagram of the electrical setup for the discharge and the power-interruption experiment. We used a parallel connexion of three power supply units (made by Heinzinger and Knrr-Heinzinger, respectively), which were protected with a block of high-power diodes (type SKN/10018 by Semikron). The technical data of the power supplies can be found in table 4 . In previous experiments, we used the 


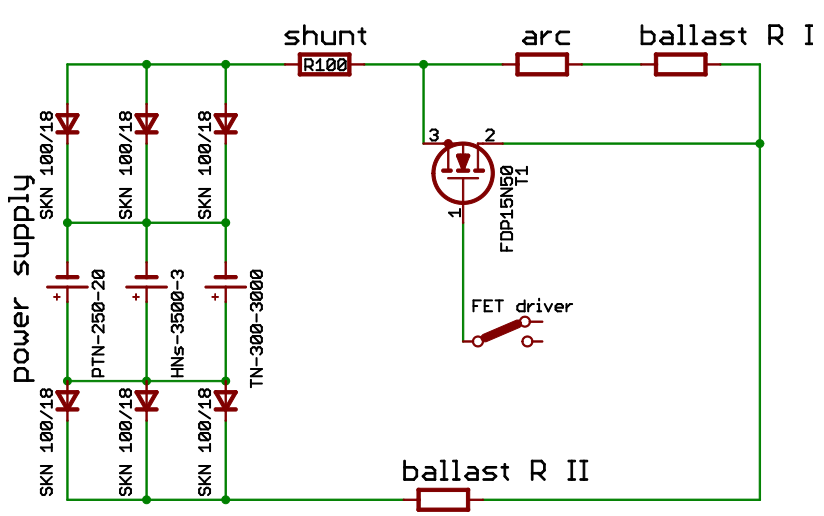

Figure 5. Block diagram of the electrical setup.

Table 4. Technical data of the power supply units.

\begin{tabular}{lcc}
\hline Model & $U_{\max }(\mathrm{V})$ & $I_{\max }(\mathrm{A})$ \\
\hline HNs 3500-3 & 3500 & 3 \\
TN 300-3000 & 300 & 10 \\
PTN 250-20 & 250 & 20 \\
\hline
\end{tabular}

high-voltage power supply to ignite the discharge. However, facing an increasing amount of components being sensitive to $\mathrm{HV}$, we developed an alternative method of ignition, which we briefly describe in section 5.4. This made the usage of HV obsolete. The remaining two supply units were able to deliver a maximum current of $30 \mathrm{~A}$ at $250 \mathrm{~V}$.

We used digital multimeters connected to a PC to monitor the voltage and the current of the discharge. For measuring the current we used a precision shunt (type RUG-Z). There were two additional sets of ballast resistors. The first set (labelled R-I in figure 5) consisted of a parallel connexion of 11 resistors (type BRQ), each having a resistance of $100 \Omega$. Both the RUG-Z and the BRQs are planar resistors manufactured by Isabellenhtte, and feature an extremely low inductivity. The second set of ballast resistors (R-II) consisted of a parallel connexion of conventional wire-wound resistors. The actual combination and thus the resulting resistance could be chosen by a set of relays.

We achieved a fast power-interruption for the relaxation experiments by short-circuiting the arc and the R-I with a pulser unit (see figure 5) employing a fast Power-MOSFET (type FDP-15N50 by Fairchild). R-I ensured a mimimum load of about $10 \Omega$ (the arc itself had a resistance of about $1 \Omega$ when operated continuously with $12.5 \mathrm{~A}$ ), which the MOSFET could short-circuit fast. The MOSFET was driven by a special chip (MAX-5048 by Maxim) to achieve short switching times. We prevented the appearance of inductive voltage spikes by keeping the leads between the discharge and the pulser unit as short as possible and by using the planar low-inductance BRQ resistors. We used a digital transient recorder (Tektronix DSA-602A) with an analogue bandwidth of $1 \mathrm{GHz}$ to monitor the power-interruption process and to adjust trigger pulses and gate widths. Using this setup we reached interruption times less than $250 \mathrm{~ns}$ (current drop to less than $10 \%$ of the initial value).

Figure 6 shows the propagation of the trigger pulses. The master trigger was created by a delay generator (Stanford Research DG-535), which was controlled by a PC. The delay

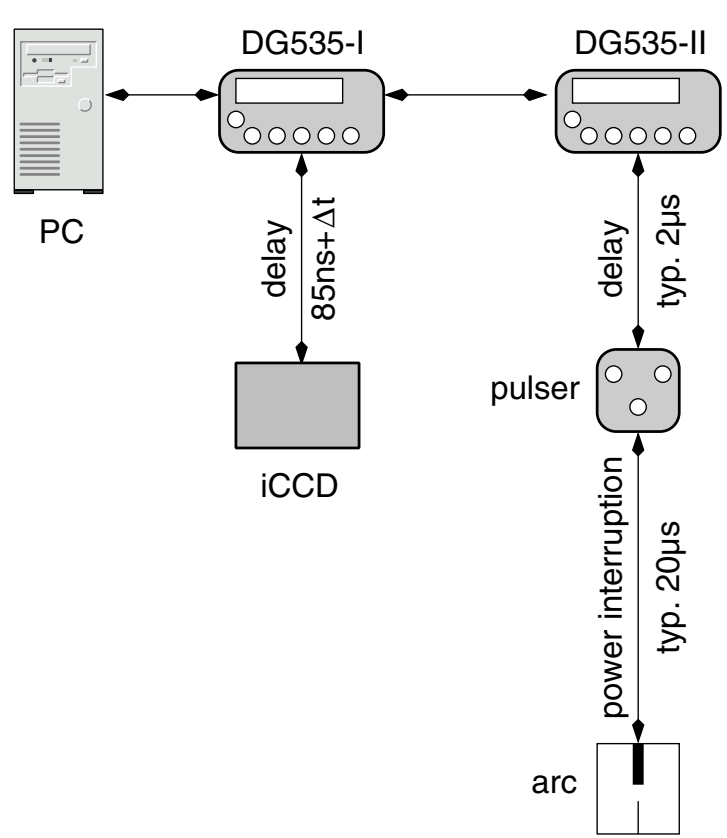

Figure 6. Trigger pulses for the relaxation experiments.

generator prepared the iCCD for exposure (which takes a minimum time of $85 \mathrm{~ns}$ for technical reasons), waited for another time interval $\Delta t$ and then exposed the CCD for the chosen time interval. The main trigger also reached a second delay generator (type DG-535), which inserted another adjustable delay (typically $2 \mu \mathrm{s}$ ). Then the second DG-535 generated a trigger pulse with a gate width of typically $20 \mu \mathrm{s}$, which we used for the pulser unit to initiate the relaxation experiment. After $20 \mu$ s of power interruption the MOSFET was switched off again, so the arc continued burning.

This setup allowed us to choose the exposure time independently of the power interruption by setting appropriate values for $\Delta t$. The additional delay of $2 \mu \mathrm{s}$ was long enough to obtain some iCCD frames before the actual relaxation experiment (still during continuous operation). Because $20 \mu \mathrm{s}$ were far too short to extinguish the arc (see timescale for recombination in table 2), the experiment could be carried out repeatedly, and the iCCD could collect photons on-chip to increase the $S / N$ ratio before the readout took place. By observing the plasma emission, we found that the arc got back to its continuous state in less than $1 \mathrm{~ms}$. Therefore, we used repetition frequencies between 100 and $200 \mathrm{~Hz}$ in this work. A typical measurement took between 1 and $5 \mathrm{~s}$, averaging over a maximum of 1000 single events. Thereby we set the exposure time for the iCCD to $200 \mathrm{~ns}$ to be able to resolve the relaxation process temporally. As a standard diagnostic line for the relaxation experiment we used the Ar I line at $7147 \AA$ (see table 1).

Data processing for the relaxation experiment was similar to line evaluation in classical emission spectroscopy as described in section 3. For each frame we integrated the line spectrally, subtracted the continuum and performed an Abel inversion. This provided us with temporally and spatially resolved line emission data for the relaxation process. We show an example of these data in figure 7 using a pseudo-colour representation for the measured LEC. The measured data for 


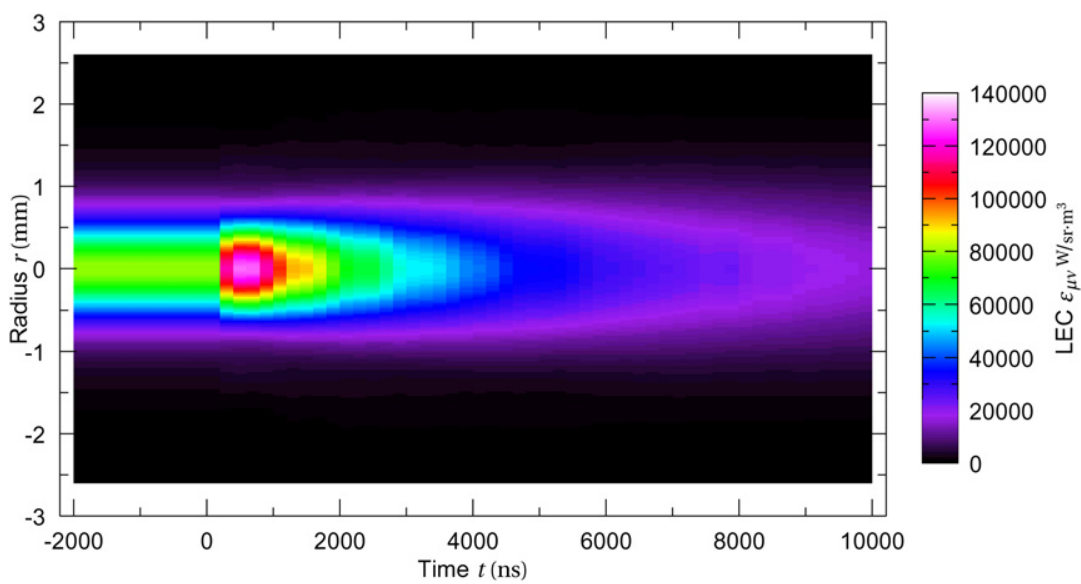

Figure 7. Spatially and temporally resolved relaxation process following a power interruption. The measurement was carried out for the first sheath $(z=50 \mu \mathrm{m})$ in front of the cathode at $I=12.5 \mathrm{~A}$. The temporal gate width for the exposure of the iCCD was $200 \mathrm{~ns}$.

$t \leqslant 0$ ns are averaged to increase the $S / N$ ratio. Following the power-interruption at $t=0 \mathrm{~ns}$ we see a sudden increase in line emission, which reaches a maximum at $t=800 \mathrm{~ns}$ and is followed by a slower decrease. A horizontal line in figure 7 corresponds to the curve progression sketched in figure 2 .

From the data presented in figure 7 we obtained the relaxation quotient $\Theta$ by taking the frame which showed the maximum LEC and dividing it by the averaged data frames for $t \leqslant 0$ ns. Having determined $\Theta$ we calculated $\beta$ according to (13). Results for various cathode distances $z$ are presented along with the other measured plasma parameters in section 6.

\subsection{Ignition of the discharge}

In previous works [1-3] we ignited the discharge either by applying high-voltage to reach an electric breakdown between the electrodes, or by initiating a glow discharge at low pressure and a subsequent transition to the arc discharge by raising current and pressure. Both methods were not applicable with the experimental setup used in this work. The MOSFET we used for power-interruption measurements had a maximum reverse voltage of $500 \mathrm{~V}$. Devices with a higher voltage exist, but these usually have slower switching times. Apart from that, the design of a smaller discharge vessel, which is advantageous in many other respects, led to small isolation gaps in some places, so that independently of the pressure used for ignition the discharge would not start between the electrodes.

Thus we developed a new method of ignition using our pulsed Nd: YAG laser system. By focusing the laser in the centre between the electrodes, we created a laser-induced optical breakdown in atmospheric argon. Once this small plasma was produced, we reached the electrical breakdown and the subsequent arc discharge by applying relatively low voltages between 200 and $250 \mathrm{~V}$.

We found that this method of ignition is favourable for several reasons.

(i) There was no need for high voltage.

(ii) The discharge always ignited between the electrodes and touched neither the electrode holders nor the housing.

(iii) The ignition was possible at atmospheric pressure, so no evacuation was required between subsequent ignitions.

(iv) The ignition was fast and reproducible. (v) The strain on the cathode was smaller compared with HV ignition.

\section{Results and discussion}

\subsection{Electron density, electron temperature and temperature ratio}

Figures 8 and 9 show the spatially resolved electron densities and electron temperatures, respectively, which we obtained for the diffuse mode at $I=12.5 \mathrm{~A}$. We determined these parameters using passive emission spectroscopy as described in section 3. Both $n_{\mathrm{e}}$ and $T_{\mathrm{e}}$ show the expected strong monotone axial and radial gradients. We found the highest temperature and the highest electron density in the centre of the discharge in the first sheath in front of the cathode. The maximum temperature was about $13500 \mathrm{~K}$; the maximum density was about $5 \times 10^{22} \mathrm{~m}^{-3}$.

Many publications (theoretical as well as experimental) deal with high-current and cascaded arcs, but only a few deal with low-current free-burning arcs; hence, there is only limited available data to compare our results. As expected, the measurements presented in this work are consistent with the results we obtained earlier in our group with a different version of the discharge vessel [52]. Our colleagues at the University of Bochum work on discharges with basically similar parameters and observe the same diffuse mode [53] as in our work. Their spectroscopic investigations yield values for $n_{\mathrm{e}}$ and $T_{\mathrm{e}}$ comparable to our values [24,54], and their simulated electron temperature matches closely, too.

The calculations in [55] including effects of ambipolar diffusion predict electron densities which are more than an order of magnitude lower than the values we have measured. On the other hand, the same paper contains LTE calculations that match our data much better. Thus, we suppose that ambipolar diffusion is not an important effect in the plasma region around the cathode of our discharge. The results in [56] show the same deviations from our experimental data: both $n_{\mathrm{e}}$ and $T_{\mathrm{e}}$ are calculated too low (the electron densities again by more than an order of magnitude). A theoretical model that yields results in good compliance with our measurements for $n_{\mathrm{e}}$ and $T_{\mathrm{e}}$ can be found in $[57,58]$. 


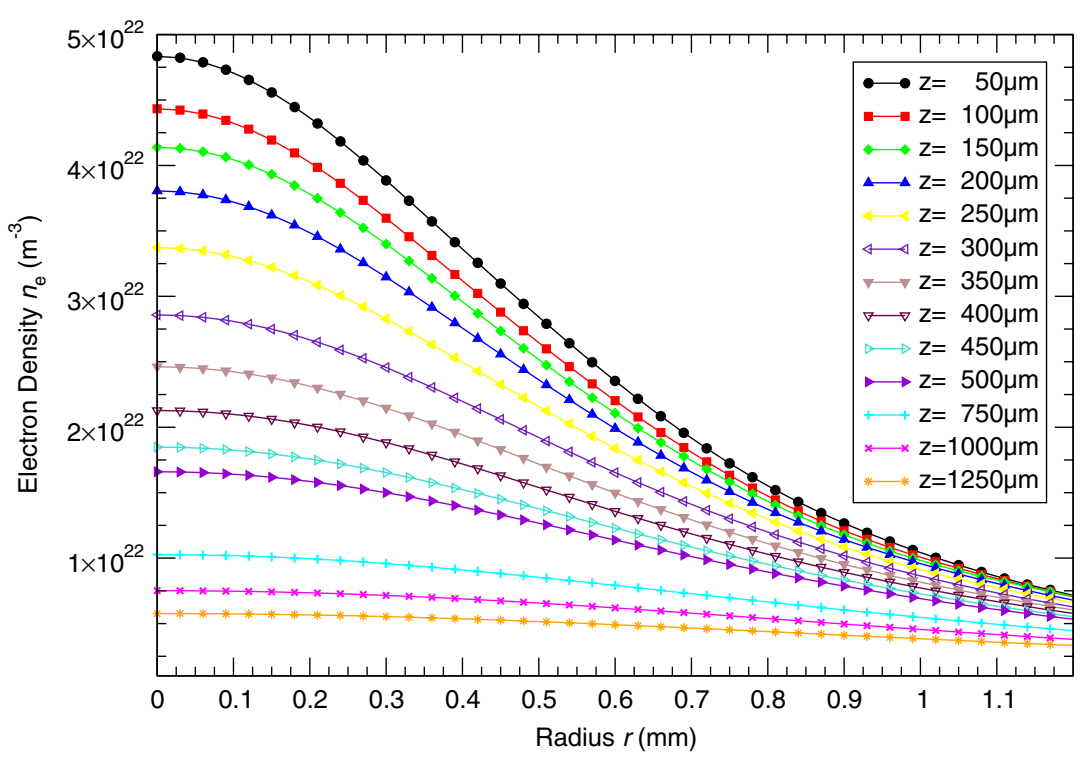

Figure 8. Spatially resolved electron densities for the diffuse mode with $I=12.5$ A.

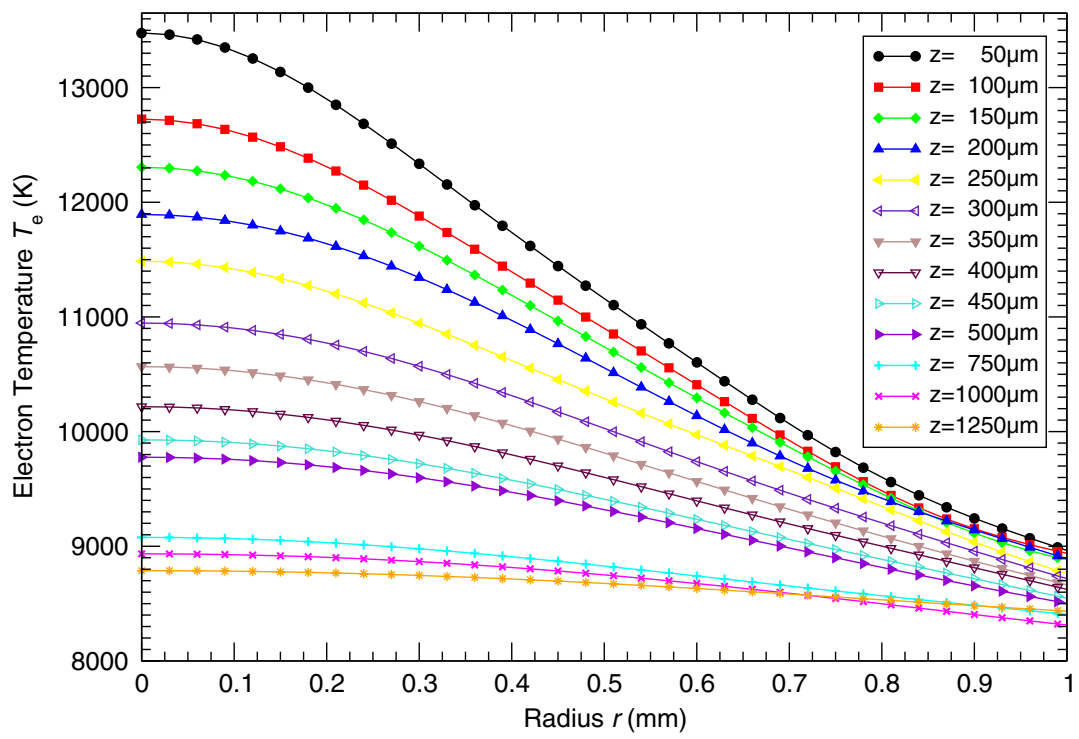

Figure 9. Spatially resolved electron temperatures for the diffuse mode with $I=12.5 \mathrm{~A}$.

Figure 10 shows the spatially resolved temperature ratio $\beta:=T_{\mathrm{e}} / T_{\mathrm{g}}$, which we determined using the relaxation method as described in section 4. $\beta$ shows axial and radial gradients with the maximum value in the arc centre in front of the cathode, similarly to $n_{\mathrm{e}}$ and $T_{\mathrm{e}}$. For distances from the cathode greater than $250 \mu \mathrm{m}$ the intensity jump of the emission line was still there, but it was very weak leading to noisy data. For $z>500 \mu \mathrm{m}$ a small jump could still be seen, but a proper evaluation of the data became impossible. To obtain a better overview of the temperature ratio in the cathode region, we show the spatial distribution of $\beta$ using a pseudo-colour mapping in figure 11. Additional supporting points were calculated by linear interpolation, respectively. Figure 12 contains the corresponding image for $T_{\mathrm{g}}$. Moreover, we present the centre line data for $n_{\mathrm{e}}$ and $T_{\mathrm{e}}$ in figure 13 .

We note that the minimum of $T_{\mathrm{g}}$ at $(z=150 \mu \mathrm{m}, r=0)$, that can be seen in figure 12 , is caused by a temperature shift of merely $200 \mathrm{~K}$ which is not beyond the uncertainty of the measurements. However, we are preparing a subsequent paper which will contain measurements obtained in the blue core mode, where a larger temperature ratio leads to a better $S / N$ ratio and thus allows us to comment on structures such as this on a more solid basis.

There are only very few publications available that provide an experimental determination of $\beta$ in arc discharges suitable for comparison with our values. Similar values (roughly between 1 and 2) are presented in [22] for the bulk plasma of a cascaded arc. However, the bulk plasma shows a behaviour, upon parameter variation, which is completely different from what we saw in the cathode region in our experiment. In [22] a rising current leads to a rising electron density and rising electron temperatures, and thus $\beta$ reaches unity as the bulk plasma approaches LTE along with the higher collision rates. In contrast, figure 11 shows that $\beta$ indicates stronger deviations 
from LTE when approaching the cathode, even though $n_{\mathrm{e}}$ and $T_{\mathrm{e}}$ are higher in this region. We conclude that in the vicinity of the cathode processes prevail which advance deviations from LTE.

We regard this result as a consequence of the close presence of the cathodic solidbody, which is relatively cool

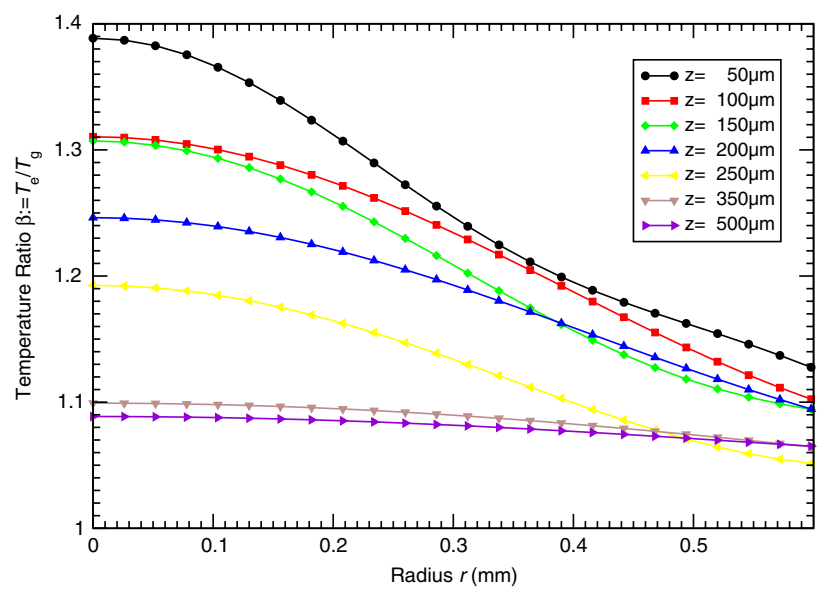

Figure 10. Spatially resolved temperature ratio for the diffuse mode with $I=12.5$ A. in comparison with the electrons leaving the space charge zone into the plasma. When approaching the cathode, the gas temperature drops to the temperature of the cathode. A similar effect occurs when approaching the wall of a wall-stabilized arc [56]. Furthermore, we note that the axial size of this region with $T_{\mathrm{e}} \neq T_{\mathrm{g}}$ appears to be larger than the size usually assumed in theory (e.g. $100 \mu \mathrm{m}$ in [56] or $20 \mu \mathrm{m}$ in [59]).

\subsection{Underpopulation factor and electric field strength}

Having determined three plasma parameters $\left(n_{\mathrm{e}}, T_{\mathrm{e}}, \beta\right)$ independently, the pLTE model presented in section 2.2 was completely determined, so we could calculate other quantities.

The first parameter to calculate was the underpopulation factor $b$. However, as already mentioned in [14, 22], the errors for $b$ are considerable because of the way the underpopulation is linked to the exponential factors in the Boltzmann distribution and the Saha-Boltzmann relation. The uncertainty can quite easily exceed $50 \%$. Thus, the values presented in figure 14 can only serve as a rough overview. On the other hand, $b$ shows strong dependences on both $z$ and $r$, so that it still abides a monotone curve progression. In spite of these problems, the values we obtained for $b$ seem to

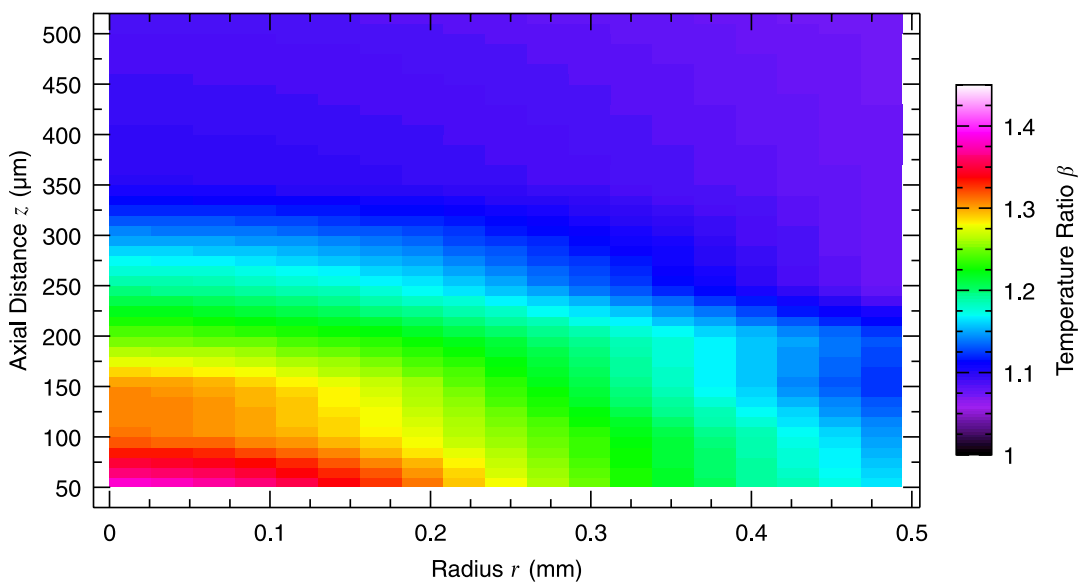

Figure 11. The 3D spatially resolved temperature ratio for the diffuse mode with $I=12.5 \mathrm{~A}$ using a pseudo-colour representation.

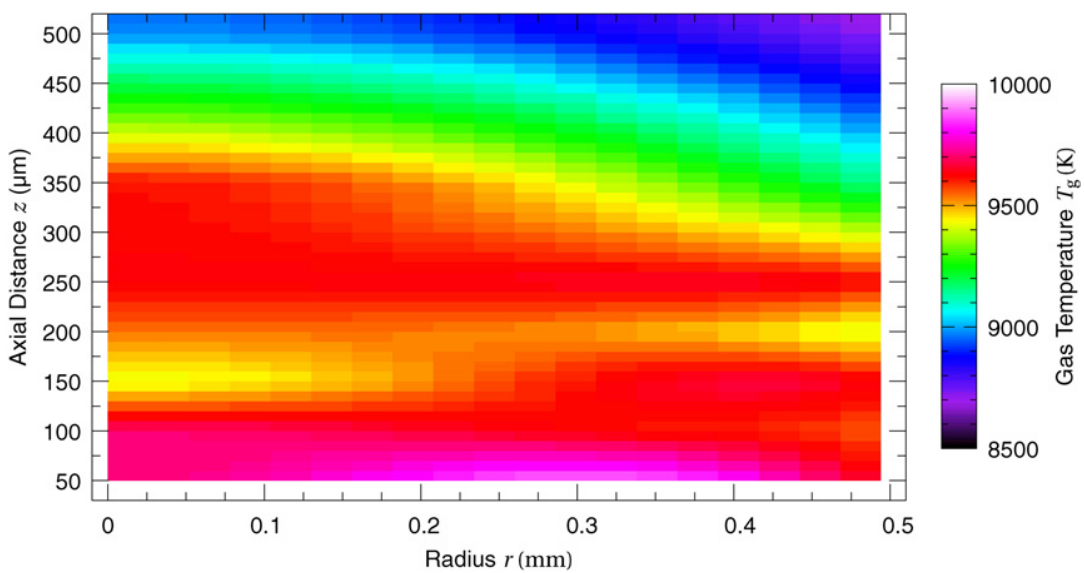

Figure 12. The 3D spatially resolved gas temperature for the diffuse mode with $I=12.5 \mathrm{~A}$ using a pseudo-colour representation (calculated from the data shown in figures 9 and 10). 


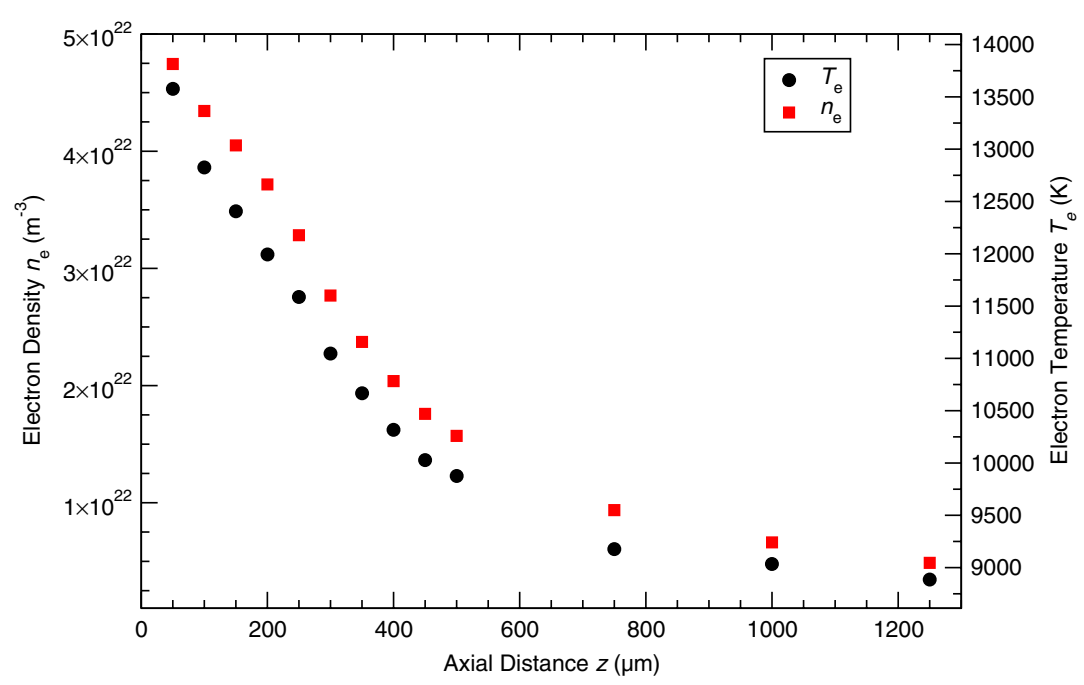

Figure 13. Measured centre line values for $n_{\mathrm{e}}$ and $T_{\mathrm{e}}$ from figures 8 and 9.

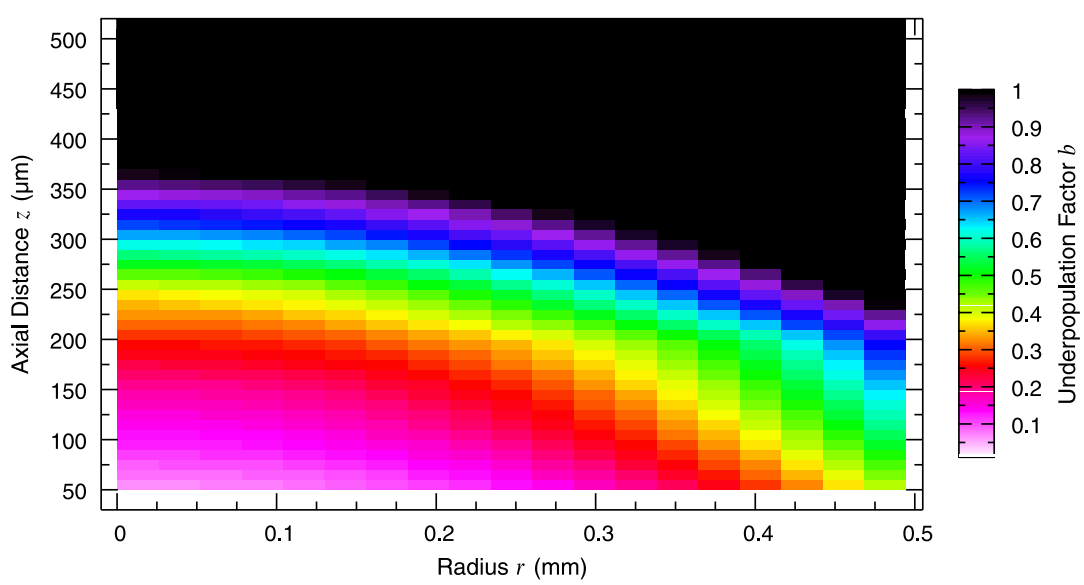

Figure 14. The 3D spatially resolved underpopulation factor for the diffuse mode with $I=12.5 \mathrm{~A}$ using a pseudo-colour representation (calculated from the data shown in figures 8-10).

be reasonable when comparing them with experimental data in [22] or calculated data in [60].

Furthermore, we could calculate the electric field strength $E$ from the measured values of $n_{\mathrm{e}}, T_{\mathrm{e}}$ and $\beta$ using a formula from [61]

$$
\frac{\beta-1}{\beta}=\frac{m_{\mathrm{Ar}}}{9 m_{\mathrm{e}}}\left(\frac{\lambda_{\mathrm{e}} e \vec{E}}{k_{\mathrm{B}} T_{\mathrm{e}}}\right)^{2},
$$

where $\lambda_{\mathrm{e}}$ is the mean free path length. We consider collisions with electrons, Ar I, Ar II and Ar III, and set according to [16]

$$
\lambda_{\mathrm{e}}:=\left(n_{\mathrm{e}}\left\langle Q_{\mathrm{ee}}\right\rangle+\sum_{i=0}^{2} n_{i}\left\langle Q_{\mathrm{e} i}\right\rangle\right)^{-1} .
$$

We obtain the values for neutral argon $\left(\left\langle Q_{\mathrm{e} 0}\right\rangle\right)$ by cubic spline interpolation of the data published in [15], whereas we calculate the values for ions ( $\left\langle Q_{\mathrm{e} 1}\right\rangle$ for $\operatorname{Ar}$ II and $\left\langle Q_{\mathrm{e} 2}\right\rangle$ for $\operatorname{Ar}$ III) according to [16, chapter II, section 8]. Further, we assume $\left\langle Q_{\mathrm{ee}}\right\rangle$ to be equal to $\left\langle Q_{\mathrm{e} 1}\right\rangle$ (following [16] again). Figure 15 displays the spatial distribution of the resulting electrical field. We present an overview of the centre line data for $\beta$ and $E$ in figure 16.
We note that (14) yields only a rough estimate for $E$. According to [62] it contains an uncertainty factor $\alpha \in[0.75$ 1.38], which we set equal to unity. Moreover, the steep gradients along the arc radius may cause additional deviations. These can only be taken into account using a more refined model of the discharge. The reader should we well aware of these inaccuracies when interpreting the values for $E$ presented in figures 15 and 16 .

\section{Conclusion and prospects}

In this work, we presented the results of pLTE plasma diagnostics in the near-cathode region of a low-current freeburning arc operated in argon under atmospheric pressure in the diffuse mode of arc attachment. We used classical (passive) emission spectroscopy to measure the electron density $n_{\mathrm{e}}$ and the electron temperature $T_{\mathrm{e}}$ from continuous and line emissions. We extended the relaxation method developed by [32] to determine the ratio $\beta$ between $T_{\mathrm{e}}$ and the gas temperature $T_{\mathrm{g}}$. By employing a fast intensified CCD detector for the relaxation experiment we were able to measure $\beta$ with a high temporal resolution and a high dynamic range. 


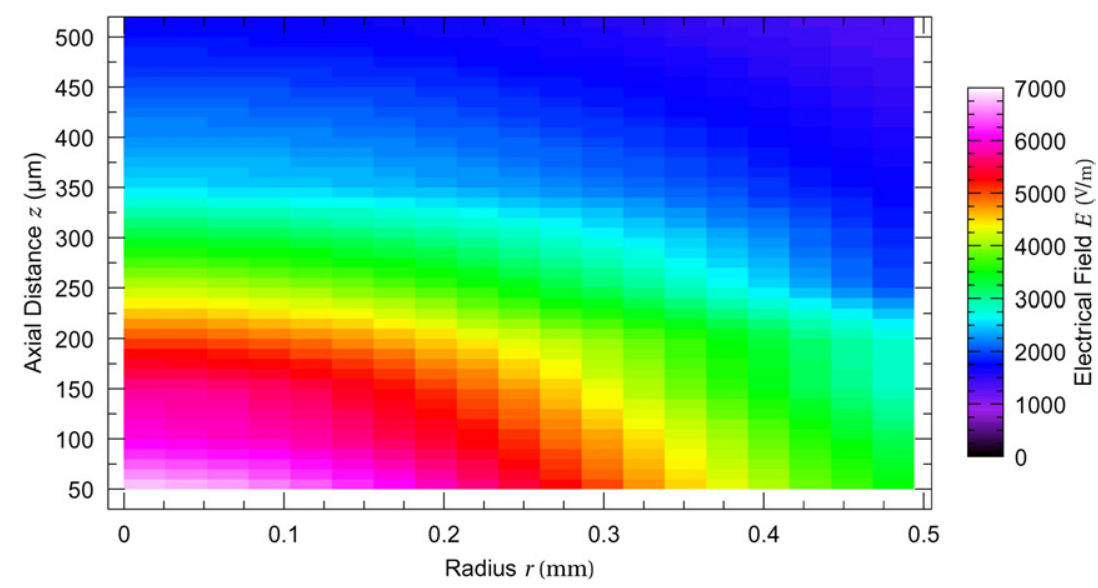

Figure 15. The 3D spatially resolved electrical field for the diffuse mode with $I=12.5 \mathrm{~A}$ using a pseudo-colour representation (calculated from the data shown in figures 8-10).

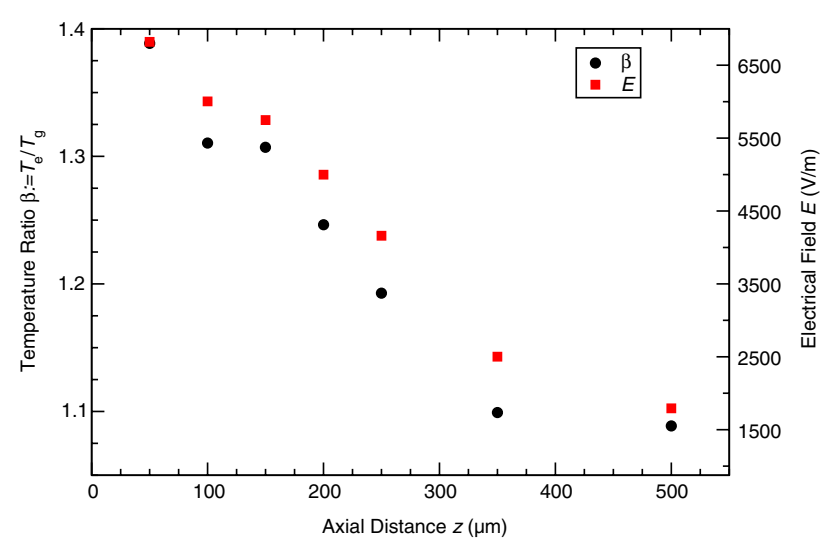

Figure 16. Measured centre line values for $\beta$ and $E$ from figures 11 and 15 .

Using basically the same data processing and Abel inversion techniques as for the evaluation of the CCD data in passive emission spectroscopy, we were able to determine $\beta$ along with $n_{\mathrm{e}}$ and $T_{\mathrm{e}}$ in $3 \mathrm{D}$ with a spatial resolution of $50 \mu \mathrm{m}$. The three parameters completed the pLTE plasma model and allowed us to further calculate quantities such as the underpopulation factor $b$ and the electric field strength $E$.

The unique feature of the method we described in this paper is the possibility of measuring the spatially resolved temperature ratio $\beta$ (and hence the gas temperature $T_{\mathrm{g}}$ ). In a subsequent paper we will present an in-depth analysis of the pLTE plasma state in the near-cathode region for different currents and different discharge modes. The spatially resolved relaxation method will be an integral part of this work.

\section{Acknowledgments}

We thank Osram for providing us with the electrode material, K Danzmann for permitting the completion of this work in his institute and $\mathrm{M}$ Heurs for a critical reading of the manuscript.

\section{References}

[1] Reiche J, Könemann F, Mende W and Kock M 2001 J. Phys. D: Appl. Phys. 34 3177-84
[2] Kühn G, Könemann F and Kock M 2002 J. Phys. D: Appl. Phys. 35 2096-104

[3] Könemann F, Kühn G, Reiche J and Kock M 2004 J. Phys. D: Appl. Phys. 37 171-9

[4] Richter J 1968 Radiation of Hot Gases Plasma Diagnostics ed W Lochte-Holtgreven (Amsterdam: North-Holland)

[5] Griem H R 2005 Principles of Plasma Spectroscopy (Cambridge: Cambridge University Press)

[6] Schlender B and Traving G 1965 Z. Astrophys. 61 92-4

[7] Traving G, Baschek B and Holweger H 1966 Abhandlungen aus der Hamburger Sternwarte vol VIII 3-25

[8] Wiese W L, Brault J W, Danzmann K, Helbig V and Kock M 1989 Phys. Rev. A 39 2461-71

[9] Vujnović V and Wiese W L 1992 J. Phys. Chem. Ref. Data 21 919-39

[10] Pellerin S, Musiol K, Dzierzega K and Chapelle J 1997 J. Quant. Spectrosc. Radiat. Transfer 57 359-76

[11] Olsen H N 1963 J. Quant. Spectrosc. Radiat. Transfer 3 $59-76$

[12] Larenz R W 1951 Z. Phys. 129 343-64

[13] Richter J 1971 10th Int. Conf. on Phenomena in Ionized Gases (Oxford, UK) pp 37-58

[14] Richter J 1980 J. Phys. Coll. C $3217-23$

[15] Devoto R S 1967 Phys. Fluids 10 354-64

[16] Mitchner M and Kruger C H 1973 Partially Ionized Gases (New York: Wiley)

[17] Sansonetti J E, Martin W C and Young S L 2004 NIST Handbook of Basic Atomic Spectroscopic Data http://physics.nist.gov/PhysRefData/Handbook/index.html

[18] Ralchenko Y, Kramida A E and Reader J 2005 NIST Physical Reference Data http://physics.nist.gov/PhysRefData/ASD/

[19] Bässler P 1978 Spektroskopische und interferometrische Untersuchungen an Lichtbogenplasmen PhD Thesis Universität Hannover

[20] Bässler P and Kock M 1980 J. Phys. B: At. Mol. Phys. 13 1351-61

[21] Nick K P 1982 Analyse des Plasmazustandes und Bestimmung atomarer Konstanten in einem Argon-Kaskadenbogen $P h D$ Thesis Universität Kiel

[22] Nick K P, Richter J and Helbig V 1984 J. Quant. Spectrosc. Radiat. Transfer 32 1-8

[23] Pokrzywka B, Musioł K, Pellerin S, Pawelec E and Chapelle J 1996 J. Phys. D: Appl. Phys. 29 2644-9

[24] Redwitz M, Langenscheidt O and Mentel J 2005 J. Phys. D: Appl. Phys. 38 3143-54

[25] Kühn G 2005 Aktive und passive Plasmaspektroskopie im Kathodenbereich eines freibrennenden Lichtbogens $P h D$ Thesis Universität Hannover (Berlin: Logos) ISBN 3-8325-1118-0

[26] Schulz-Gulde E 1970 Z. Phys. 230 449-59 
[27] Pokrzywka B, Pellerin S, Musiol K and Chapelle J $1999 \mathrm{~J}$. Phys. D: Appl. Phys. 32 1665-70

[28] Hofsaess D 1978 J. Quant. Spectrosc. Radiat. Transfer 19 339-52

[29] Schnehage S E, Kock M and Schulz-Gulde E 1982 J. Phys. B: At. Mol. Phys. 15 1131-5

[30] Wilbers A T M, Kroesen G M W, Timmermans C J and Schram D C 1991 J. Quant. Spectrosc. Radiat. Transfer 45 1-10

[31] Leskov L V and Savin F A 1961 Sov. Phys.-Usp. 3 912-27

[32] Gurevich D B and Podmoshenskii I V 1963 Opt. Spectrosc. (USSR) 15 319-22

[33] Aleksandrov V Y, Gurevich D B and Podmoshenskii I V 1965 Opt. Spectrosc. (USSR) 18 107-10

[34] Alexandrov V Y, Gurevich D B and Podmoshenskii I V 1967 Opt. Spectrosc. (USSR) $23282-6$

[35] Aleksandrov V Y, Gurevich D B and Podmoshenskii I V 1968 Opt. Spectrosc. (USSR) 25(5) 378-80

[36] Kafrouni H, Bagneux J M, Gleizes A and Vacquie S 1979 J. Quant. Spectrosc. Radiat. Transfer 21 457-73

[37] Gleizes A 1981 Physica C 111 386-96

[38] Gleizes A, Kafrouni H, Dang Duc H and Maury C $1982 \mathrm{~J}$ Phys. D: Appl. Phys. 15 1031-45

[39] Bydder E L and Miller G P 1988 Spectrochim. Acta B 43 819-29

[40] Fey F H A G, Stoffels W W, van der Mullen J A M, van der Sijde B and Schram D C 1991 Spectrochim. Acta B 46 885-900

[41] van der Mullen J A M, Benoy D A, Fey F H A G, van der Sijde B and Vlcek J 1994 Phys. Rev. E 50 3925-34

[42] van der Mullen J A M and de Regt J M 1996 Fres. J. Anal. Chem. 355 532-7

[43] Bouaziz M, Gleizes A and Razamfinimanana M 1998 J. Appl. Phys. 84 4128-36

[44] Shindo H, Imazu S and Inaba T 1981 J. Quant. Spectrosc. Radiat. Transfer 25 77-81

[45] Kafrouni H 1979 Physica C 98 100-12

[46] Press W H, Flannery B P, Teukolsky S A and Vetterling W T 1989 Numerical Recipes in Pascal (Cambridge: Cambridge University Press)
[47] Czack G, Kirschstein G, Kurtz W and Stein F 1993 W/Tungsten (Gmelin Handbook of Inorganic and Organometallic Chemistry) ed W Huisel et al (Berlin: Springer) A4 System Number 54

[48] Tanaka M, Ushio M, Ikeuchi M and Kagebayashi Y 2005 J. Phys. D: Appl. Phys. 38 29-35

[49] Pretzler G 1991 Z. Naturf. 46 a 639-41

[50] Pretzler G, Jäger H, Neger T, Philipp H and Woisetschläger J 1992 Z. Naturf. 47 a $955-70$

[51] Kock M and Richter J 1969 Ann. Phys. Lpz. 24 30-7

[52] Könemann F 2000 Emissionsspektroskopie im kathodennahen Bereich eines Lichtbogens $P h D$ Thesis Universität Hannover, http://edok01.tib.uni-hannover.de/ edoks/e01dh01/3281269421.pdf

[53] Dabringhausen L, Langenscheidt O, Lichtenberg S, Redwitz $\mathrm{M}$ and Mentel J 2005 J. Phys. D: Appl. Phys. 38 3128-42

[54] Redwitz M 2003 Charakterisierung des Plasmas nahe der Elektroden für Hochdruck-Plasmalampen durch spektroskopische Messungen PhD Thesis Ruhr-Universität Bochum Berichte aus der Elektrodenforschung vol 8 (Berlin: Tenea) ISBN 3-86504-078-0

[55] Sansonnens L, Haidar J and Lowke J J 2000 J. Phys. D: Appl. Phys. 33 148-57

[56] Benilov M S 1999 IEEE Trans. Plasma Sci. 27 1458-63

[57] Rethfeld B, Wendelstorf J, Klein T and Simon G 1996 J. Phys. D: Appl. Phys. 29 121-8

[58] Wendelstorf $\mathbf{J} 2000 \mathrm{Ab}$ initio modelling of thermal plasma gas discharges $P h D$ Thesis Technische Universität Braunschweig, http://www.biblio.tu-bs.de/ediss/ data/20010215a/20010215a.html

[59] Lowke J J, Kovitya P and Schmidt H P 1992 J. Phys. D: Appl. Phys. 25 1600-6

[60] Vlček J and Pelikán V 1990 J. Phys. D: Appl. Phys. 23 526-32

[61] Finkelnburg W and Maecker H 1956 Elektrische Bögen und thermisches Plasma In Handbuch der Physik vol XXII: Gasentladungen II ed S Flügge (Berlin: Springer) pp 306-7

[62] Maecker H 1951 Erg. d. exakt. Naturw. XXV 293-358 